\title{
Las relaciones fabricante distribuidor como elementos básicos de un modelo competitivo en el caso del cluster cerámico español. Análisis empírico de los factores moderadores.
}

\author{
J. ALBORS-GARRIGÓS ${ }^{1}$, P. MÁRQUEZ RODRÍGUEZ ${ }^{2}$, J.L. HERVÁS-OLIVER' \\ 'Dpto. de Organización de Empresas. Universidad Politécnica de Valencia. Valencia \\ ${ }^{2}$ Instituto Politécnico de Monterrey, Campus de Guanajuato, Mexico.
}

\begin{abstract}
La industria cerámica, como otras industrias tradicionales, se encuentra inmersa en un contexto de constantes cambios marcado por una importante competencia a nivel mundial. La función distribución no es una excepción y viene cobrando un rol cada vez más relevante en la economía globalizada. Por otra parte, la proliferación de información y su fácil acceso sobre productos y servicios esta obligando a los distribuidores e intermediarios tradicionales a buscar nuevas formas de relacionar a compradores y vendedores a través de mecanismos que garanticen la completa satisfacción del usuario final. Este trabajo tiene como objeto explorar la influencia y el rol de los canales de distribución en la creación de valor en el sector cerámico español. En este sentido se analizan tres factores moderadores en el modelo: Actividades de orientación al mercado; intercambio de información estratégica con fabricantes; y servicios complementarios ofrecidos al cliente final.
\end{abstract}

Palabras clave: cadena de valor, relaciones fabricante-distribuidor, sector cerámico español, martketing relacional.

Relationships manufacturer distributor as key competitive elements in the case of the spanish tile ceramic cluster. Empirical analysis of the moderating factors.

The tile ceramic industry, as other traditional industries, is immersed in a context of constant changes driven by relevant competition world-wide. The distribution function is not an exception and is playing an increasing role in a global economy. On the other hand, the abundance of information and easy access to products and services is forcing distributors and traditional intermediaries to search new forms to relate with buyers and salesmen through mechanisms which guarantee the final end user complete satisfaction. This paper objective is exploring the influence and role of distribution channels in the creation of value in the case of Spanish tile ceramic industry. In this direction three moderating factors in the model have been analyzed: market oriented activities; strategic exchange of information with manufacturers; and offer of complementary services by distributors to final clients.

Keywords: value chain, manufacturer-distributor relationships, relational marketing.

\section{INTRODUCCIÓN}

La literatura académica tradicional ha analizado, casi exclusivamente, las relaciones fabricante distribuidor desde la perspectiva del fabricante y ello, de forma puntual, sin analizar el impacto que las mismas pueden tener en los clientes finales. Por esta razón, el presente trabajo plantea una nueva visión identificando las aportaciones surgidas a partir de estas relaciones, así como en el valor originado por las mismas que puede ser trasmitido y percibido por el cliente final.

En este sentido, se pretende que la distribución no se siga analizando como una actividad periférica a la estrategia competitiva de los productores, sino que pueda ser considerada un canal adaptativo, flexible y enfocado a la prestación de servicios, lo cual no es posible sin una estrecha colaboración con los productores y con el resto de los componentes de la cadena.

Es por ello que el presente trabajo se ha desarrollado bajo la perspectiva del distribuidor haciendo especial énfasis en los efectos predecesores de creación de valor para el cliente final y que puedan ser motivados a través de la influencia de las relaciones fabricante-distribuidor.

\section{OBJETIVOS}

El presente artículo analiza la relación que se establece entre los fabricantes y distribuidores de azulejos españoles, una relación del tipo proveedor-distribuidor. El objetivo principal es desarrollar un modelo que permita analizar el vínculo existente entre el nivel de relación observado entre estos dos agentes y tres factores que consideramos como predecesores del valor ofrecido al cliente final: orientación al mercado por parte de los distribuidores, intercambio de información estratégica con fabricantes y servicios complementarios ofrecidos al cliente.

Este artículo se ha organizado del siguiente modo. Primero, se analiza el estado de arte relativo a relaciones entre fabricantes y distribuidores y la creación de valor en la distribución. En segundo lugar se plantean las hipótesis relativas a los factores que influyen en esta prestación de valor. Posteriormente, se describe someramente el sector de estudio, la metodología seguida y el estudio realizado. Finalmente, se discuten los resultados y se plantean las conclusiones del trabajo 


\section{RELACIONES ENTRE FABRICANTES Y DISTRIBUIDORES}

Para autores como Lassar y Kerr (1), la competitividad de la empresa no depende únicamente de sus activos estratégicos internos, sino también del tipo de relaciones que es capaz de establecer con otras empresas y del alcance de éstas mismas, por lo que cada vez es más difícil defender una posición competitiva en los negocios basada en una capacidad individual dominante.

Las relaciones establecidas entre empresas en un canal de distribución, han sido estudiadas desde diferentes paradigmas y corrientes, lo que ha generado no sólo una diversidad conceptual sino terminológica al objeto de estudio (2). Una de éstas corrientes es el enfoque relacional, que ha integrado elementos del concepto tradicional del marketing (3), del marketing de servicios y del marketing de redes (4).

Dicho enfoque utiliza el concepto de relacionalismo que desde el punto de vista estratégico, considera más importante el mantenimiento de la relación como relación en sí misma y no como una serie de intercambios aislados (5). De tal forma, que una relación se torna importante hasta el punto de ser estratégica (6) y ser percibida como un activo, que por su carácter forma parte de la planificación estratégica de la empresa (7).Adicionalmente, Rowe y Barnes (8) reconocen la importancia de establecer intercambios relacionales ya que su resultado es "creador de valor" a través de un fenómeno social complejo muy costoso y difícil de imitar.

No obstante, los beneficios que pueden ser obtenidos a través del nivel de relacionalismo mantenido entre dos o más organizaciones deben ser traducidos a valor generado y entregado al cliente, lo cual es muy difícil de cuantificar debido, en gran parte, a la dimensión subjetiva e intrínseca que subyace al concepto de valor en sí mismo.

En este sentido, y dado que el presente trabajo ha sido desarrollado bajo la perspectiva del distribuidor, no es nuestro objetivo inmediato medir la percepción del cliente, necesario para determinar el valor final generado, sino que pretendemos concentramos en los efectos positivos predecesores de la creación de valor para el usuario final que pueden ser obtenidos a través de la influencia del relacionalismo.

\subsection{El rol de los canales de distribución en la creación de valor}

Anderson (9) describe el crear y compartir valor como la razón que da lugar a las relaciones de colaboración entre proveedores y clientes. No obstante, el estudio de éste tipo de relaciones entre los actores mencionados aún se encuentra en su etapa formativa y algunos autores (10) (11), consideran que existe una falta de conocimiento en este campo, especialmente en las áreas relacionadas directamente con el desarrollo, gestión y efectividad de las relaciones al interior de dichos canales, así como la medición del valor generado. Teniendo en cuenta dichos antecedentes, Simpson, Siguaw y Baker (12) desarrollan un marco conceptual para examinar el valor que los distribuidores obtienen derivado de sus relaciones con proveedores, y específicamente proponen los determinantes y resultados de la creación de valor percibida.

El punto de partida de éste modelo es la orientación al mercado como fuerza conductora que da origen a cierto tipo de comportamientos y actividades creadoras de valor. Así las actividades de éste tipo emprendidas por los proveedores tendrán una significativa influencia en los costos, valor percibido, desempeño financiero de los distribuidores y a su vez, al interior de la relación que tiene lugar en el canal.

\subsubsection{Orientación al Mercado}

El modelo considera la orientación al mercado como un importante precursor de la creación de valor en el contexto de un canal, definida como "la cultura que en primer lugar, otorga la máxima prioridad a la creación y mantenimiento de un valor superior para el cliente, considerando igualmente los intereses de otros actores claves y, por otra parte, provee las normas de comportamiento teniendo en cuenta el desarrollo organizacional y responsabilidad de la información de mercado" (13).

Esto último es congruente con otras definiciones más amplias de orientación al mercado como la propuesta por Deng y Dart (14) que formulan la definición de orientación al mercado como la generación de una inteligencia de mercado apropiada sobre las necesidades presentes y futuras de los clientes y las habilidades relativas de las entidades competidoras para satisfacer estas necesidades; la integración y diseminación de dicha inteligencia a lo largo de los departamentos; y el diseño y ejecución coordinados de la respuesta estratégica a las oportunidades de mercado.

Es bajo esta perspectiva sobre la que establecemos que la orientación al mercado es un elemento decisivo para la creación de mayor valor agregado a los clientes, ya que son precisamente las actividades de orientación al mercado el insumo que proporcionará a los distribuidores el conocimiento necesario para establecer con relativa exactitud las características de la demanda de un mercado determinado.

Por otra parte, vinculamos este concepto igualmente con el marketing relacional porque en el contexto específico de este estudio, el sector cerámico español, es igualmente necesario un proceso de intercambio de información entre fabricantes y distribuidores ya que los primeros se dedican al desarrollo del producto mientras que los segundos son los que mayoritariamente tienen contacto con el usuario final. De esta forma, las perspectivas que planteamos son complementarias si se tiene en cuenta que las dos son necesarias para la creación de valor por lo menos en este contexto.

Esto último nos lleva a considerar otro factor predecesor a la creación de valor como es el intercambio de información estratégica con los fabricantes, ya que teniendo en cuenta la configuración del sector azulejero español, es la única forma de trasmitir los resultados de las acciones de orientación al mercado de los distribuidores y materializarlas en las mejoras y cambios pertinentes que el fabricante introduzca en los productos.

\subsubsection{El componente de servicios en la creación de valor}

Tal como lo apuntaban Simpson, Siguaw y Baker (12) la prestación de servicios constituye otro factor a tener en cuenta en los modelos que intentan explicar la creación de valor en el contexto de los canales de comercialización.

Lusch y Leczniak (15) argumentan que para el caso de los distribuidores es especialmente importante estar atentos a las necesidades cambiantes del cliente. De tal forma que contar con una fuerte orientación de servicios en este tipo de ambientes altamente competitivos no solamente mejora la evaluación de los clientes con respecto a las ofertas de productos (16), sino que también facilitan medios para construir fuertes relaciones con el cliente e incrementar la lealtad de los mismos (17). 
Para el caso de los distribuidores consideramos dentro del estudio la exploración de los servicios complementarios prestados a los clientes desde las propias tiendas tales como: adecuación de azulejo al uso, consejos, colocación, selección entre otros. Asimismo, hemos examinado con especial énfasis la prestación de servicios a través de la creación de páginas webs por parte de los distribuidores, las cuales hemos comparado finalmente con la oferta de los fabricantes.

\section{FACTORES ANALIZADOS}

Basándose en la revisión teórica, se pretende evaluar los siguientes factores: Actividades de Orientación al Mercado/ Clientes de los Distribuidores, Intercambio de Información Estratégica con Fabricantes y Servicios Complementarios y Relación con el cliente.

La orientación al mercado requiere que el distribuidor desarrolle una comprensión exhaustiva del cliente con el objetivo de crear un valor superior continuamente (18). Esto implica conocer con la mayor exactitud posible las necesidades y expectativas del mismo, generando productos y servicios que surjan como respuesta a dichas necesidades y que se presenten como soluciones a problemas e inquietudes existentes.

Adicionalmente, una relación entre fabricantes y distribuidores que trate aspectos relacionados con el negocio más allá del proceso de compra venta como la evaluación y percepción de los clientes, ejerce una influencia positiva en la motivación de los distribuidores para estudiar exhaustivamente el comportamiento de los usuarios que diariamente se dirigen a sus tiendas y adquieren determinados productos de acuerdo a ciertos parámetros y preferencias (19).

Por esta razón la primera hipótesis de partida es:

El nivel de Relación entre fabricantes y distribuidores tiene un efecto positivo sobre la realización de actividades orientadas al mercado/cliente por parte de los distribuidores.

El segundo elemento precursor de la creación de valor para el usuario final está determinado por los servicios complementarios ofertados al cliente. Algunos autores (12) identificaban, dentro de su modelo de creación de valor, un factor específico de servicio y apoyo como una de las actividades y servicios orientados al valor.

Así, se considera que los servicios complementarios representan la respuesta a la información y conocimiento obtenido de los usuarios finales y que debe incluir también cuales son los servicios e intangibles que ejercen una influencia importante sobre el poder de compra o adquisición, y sobre la decisión de comprar en una tienda determinada, aún cuando la oferta de productos sea bastante similar entre ellas.

Una relación provechosa entre fabricantes y distribuidores puede convertirse en un factor positivo para que el distribuidor amplíe, con base en el conocimiento del producto y las posibilidades del fabricante, la gama de servicios complementarios ofrecidos al cliente, no sólo con productos de calidad sino con una serie de intangibles que a larga aumentarían la confianza de los usuarios en el producto final, intangibles que cada vez cobran mayor importancia.

Por ello, la segunda hipótesis propuesta es:

El nivel de Relación entre fabricantes y distribuidores tiene un efecto positivo sobre la intensidad de la gama de los servicios complementarios ofrecidos al cliente

El tercer factor que se ha evaluado es el intercambio de información estratégica entre fabricantes y distribuidores. Dado que, en el caso que nos ocupa, los distribuidores de material cerámico adquieren los productos de los fabricantes sin efectuar ningún tipo de transformación en los mismos, es necesario recoger información a través de las actividades o mediciones dirigidas al usuario final para que posteriormente éstas sean realimentadas al fabricante quien se encarga en su totalidad del diseño y procesos relacionados con el producto.

Bajonuestra perspectiva, estos procesos seven influenciados positivamente por un intercambio de tipo relacional, donde, tanto fabricantes como distribuidores, otorguen un cierto grado de formalización a los mismos de cara a la implantación de soluciones que acerquen la demanda del cliente a la oferta de la industria y cuyo efecto predecesor para la creación de valor evaluaremos.

Es así como intentamos corroborar la relación causal entre el mayor nivel de relación entre fabricantes y distribuidores y la medición de las expectativas y necesidades del cliente por parte del distribuidor., como un elemento influyente en el valor finalmente entregado a dicho usuario.

Por esto, consideramos que otro elemento precursor del valor agregado al cliente final es el intercambio de información estratégica entre fabricantes y distribuidores y la tercera hipótesis estaría formulada así: El nivel de Relación entre fabricantes y distribuidores tiene un efecto positivo sobre la utilización de herramientas de medición de las percepciones del cliente por parte del distribuidor

\section{SECTOR DE ESTUDIO}

Hasta el momento, la cadena de valor de la fabricación de azulejos cerámicos se ha analizado desde una perspectiva limitada que incluye únicamente una estructura como la que se presenta en la figura 1.

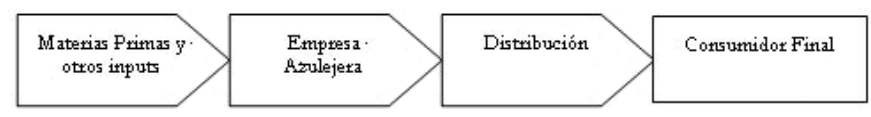

Fig. 1- Cadena de valor de los azulejos cerámicos (23).

De esta forma, se han configurado en cada componente varias tipologías de actores presentes en esta cadena, dificultando la determinación del valor agregado en cada fase desde la fabricación del producto al consumidor final.

En el cluster azulejero de Castellón se ha identificado una multiplicidad de actores, los cuales sin duda alguna, ejercen actividades que se ven reflejadas en el desempeño de la industria como un todo. Sin embargo, la gran cantidad de actores que se ven representados necesitan un análisis por separado que realice aportes para cada tipo de actor y relación por individuo, un objetivo demasiado ambicioso para un trabajo de este tipo .

Una aproximación más cercana a la realidad de la cadena de valor que estamos analizando fue elaborada por Hervás (25), que representa gráficamente las interrelaciones que se llevan a cabo entre los diferentes elementos que conforman la industria, no sólo aquellos que interactúan durante el proceso de fabricación sino los que hacen parte de las fases finales de comercialización e instituciones de soporte dentro del cluster. La figura 2 refleja la interrelación entre estos agentes. 


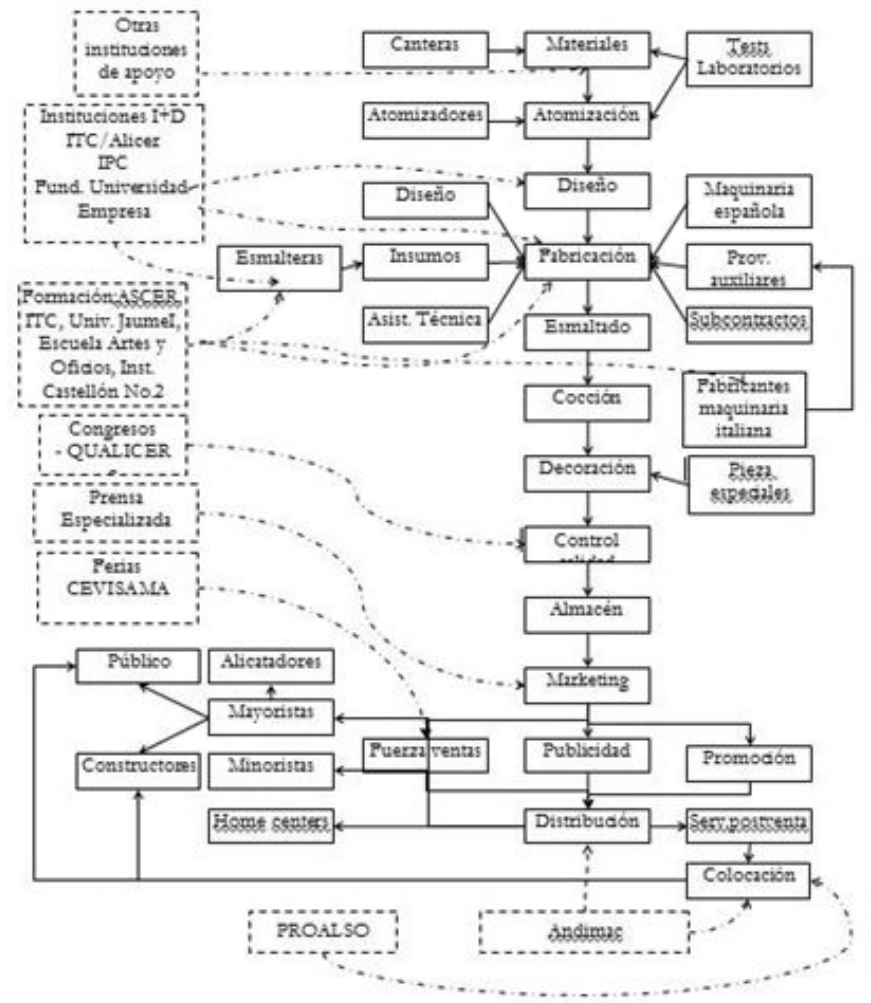

Fig. 2- Relaciones presentes en la Cadena de Valor de la industria cerámica. Modificada sobre (25).

Por esta razón si se analiza la representación gráfica de las relaciones presentes en la cadena vemos como existe una mayor densidad de relaciones en la parte inicial de la cadena, que se llevan a cabo entre fabricantes y proveedores de materias primas, maquinarias, esmalteras einstituciones de apoyo, lo que ha sido ya apuntado ppor diversos autores (20), (21), (22), (23), (24). No obstante, después de los procesos de comercialización llevados a cabo por las empresas fabricantes, vemos como las relaciones que se establecen disminuyen sensiblemente su intensidad. Esto es, se presentan principalmente relaciones de tipo comercial, de allí la importancia de profundizar en la caracterización y mejoramiento de las mismas.

\subsection{Metodología}

La metodología se ha basado en una encuesta entre una muestra representativa de 97 empresas distribuidoras de azulejos, la realización de entrevistas entre empresas distribuidoras y fabricantes líderes así como el análisis de información macroeconómica del sector.

Otra parte importante de la investigación cualitativa se ha basado en la asistencia a diversos eventos organizados por organismos del sector donde se exponía gran parte de la problemática del sector distribución y de los propios fabricantes, lo que fue un elemento importante para conocer por parte de los propios actores de la industria sus necesidades y retos en el mercado actual. Posteriormente, se analizó la información recogida en las encuestas llevando a cabo la aplicación de métodos estadísticos, tanto de carácter descriptivo como multivariante.

Los resultados obtenidos en las encuestas se reflejan en las figuras 3, 4 y 5 . En la 3 y 4 se apunta los medios y frecuencia utilizados para averiguar el nivel de satisfacción de los clientes. En la 5 la valoración de las actividades de orientación al mercado por parte de los distribuidores. Finalmente, en la 6 los servicios complementarios ofrecidos por los distribuidores a los clientes.

Se puede apreciar, como confirma el análisis multivariante, que aun son escasas las empresas que muestran un comportamiento avanzado en este campo.

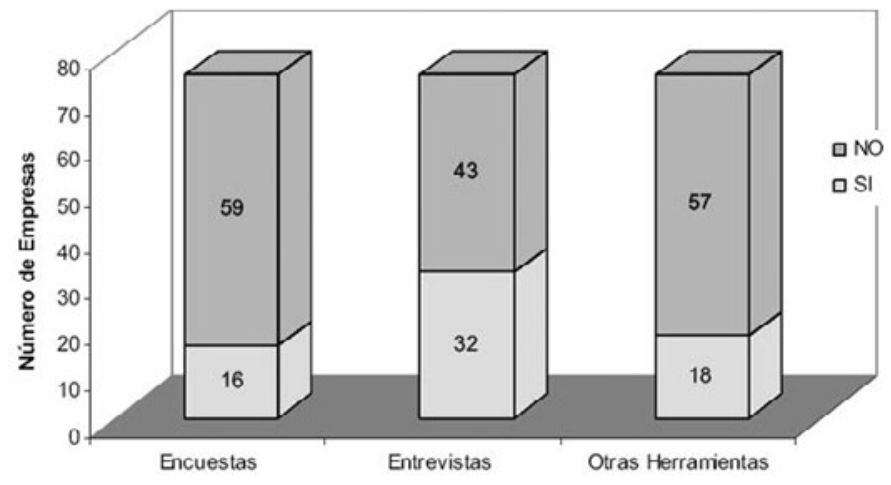

Fig. 3- Medios utilizados por los distribuidores para medir la satisfacción de los clientes.

Más de tres veces

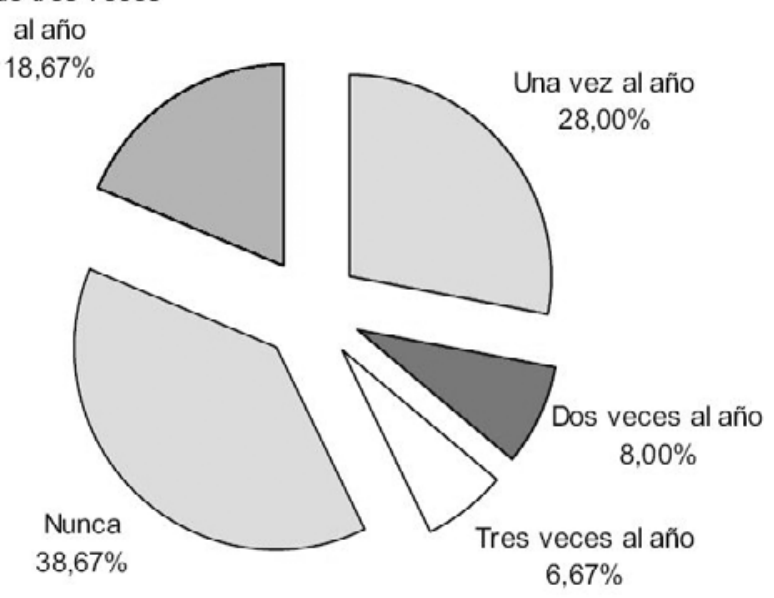

Fig. 4- Frecuencia de medición de la satisfacción de los clientes.

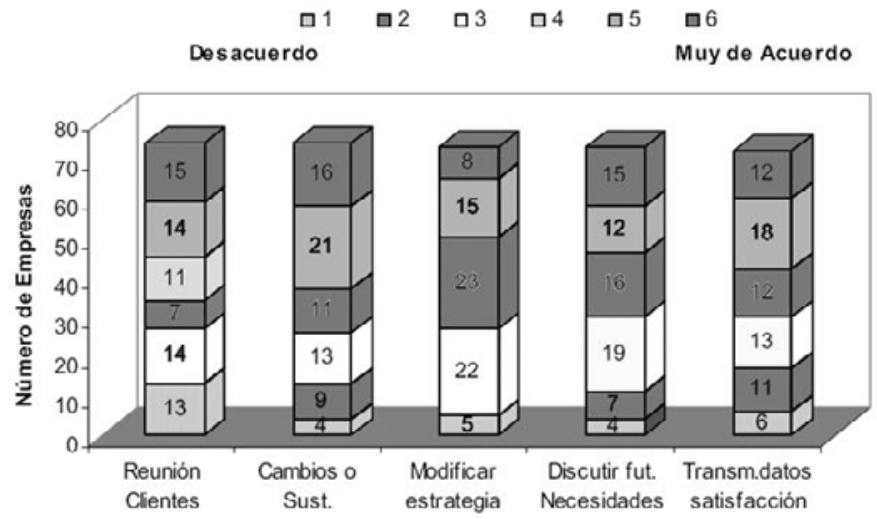

Fig. 5- Valoración en la muestra de las actividades de orientación al mercado por parte de los distribuidores. 


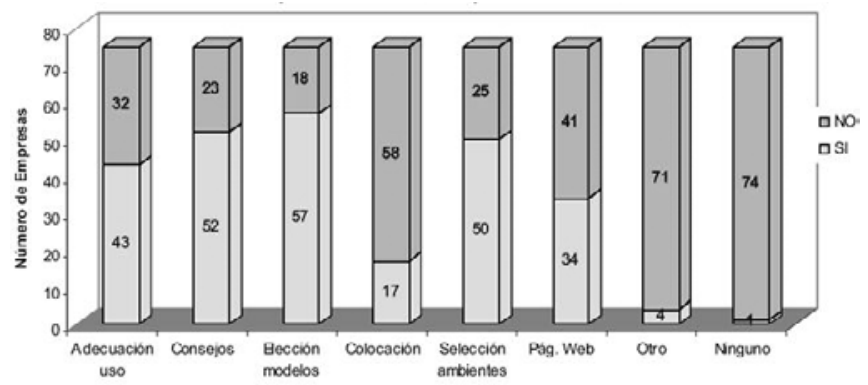

Fig. 6- Servicios complementarios ofrecidos por los distribuidores a los clientes.

\subsection{Tratamiento de datos y resultados}

Para la medición del nivel de relación entre fabricantes y distribuidores se utilizó el concepto de relacionalismo, utilizando como base los ítems que hacen parte de la escala definida por González (7), validada precisamente en el entorno de la distribución española.

No obstante, antes de proceder a efectuar la recopilación de datos se realizó un estudio de tipo cualitativo que pretendía evaluar los ítems que conformarían posteriormente la encuesta estructurada con el objetivo de conseguir una escala basada no únicamente en los fundamentos teóricos sino en la realidad del sector analizado.

A partir de los resultados del nivel de relacionalismo se clasificó la muestra, mediante un análisis cluster en tres grupos diferentes: el primero de ellos con una alta valoración de la relación con fabricantes, el segundo con una valoración media y el tercero con una valoración mínima de dicha relación.

Posteriormente, basándose en estos tres grupos se procedió al contraste de las hipótesis planteadas. La primera hipótesis que planteamos tiene que ver con la influencia del nivel de relación existente entre fabricantes y distribuidores sobre la realización de actividades claramente orientadas al mercado y que hemos reseñado en apartados anteriores.

Dado que tanto la variable dependiente: actividades de orientación al mercado, como la independiente: nivel de relacionalismo, son de tipo cualitativo, se evaluaron haciendo uso de variables de tipo nominal. Se utilizaron tablas de contingencia y pruebas de chi-cuadrado para contrastar las categorías y frecuencias de ambas variables y posteriormente validar si existían diferencias significativas que nos permitan argumentar que cada grupo se caracteriza por un grado de orientación al mercado causado al menos parcialmente por su forma de considerar las relaciones con los proveedores.

Los resultados nos indican, tal como se ha propuesto, que el grupo que se identifica con una valoración alta de la relación distribuidor- cliente es igualmente el que manifiesta llevar a cabo una mayor cantidad de actividades de orientación al mercado. En concreto, más de la mitad de los pertenecientes a este conglomerado se clasifican dentro de la categoría 3, es decir, de aquellos que valoraron la intensidad de estas actividades entre 21 y 30 .

Por su parte, los grupos 2 y 3 de distribuidores cuyo desempeño en la relación con los fabricantes es inferior, presentan, simultáneamente, una distribución diferente en el que predominan valoraciones menores de las actividades de orientación al mercado. La significatividad de estas diferencias se comprueba con la aplicación de la prueba de chi-cuadrado. Los resultados de la misma muestran que dicha significatividad corresponde al nivel $\mathrm{p}<0,01$.
Todo lo anterior nos impide rechazar nuestra hipótesis de partida y podemos afirmar que en general el nivel de relacionalismo, entre fabricantes y distribuidores, sí ejerce influencia sobre la cantidad de actividades de orientación al mercado realizadas por la empresa, lo cual se corrobora por las características y resultados observados en los conglomerados identificados. Esto es, a mayor nivel de relacionalismo por parte de los distribuidores, mayor es la disposición y realización de actividades de orientación al mercado.

Las diferencias en la intensidad de servicios complementarios ofrecidos entre los diferentes conglomerados de relacionalismo se ven corroborados por las pruebas de chi-cuadrado que presentan una significatividad al nivel $\mathrm{p}<0,05$.

Adicionalmente, las tablas de contingencia nos permiten concluir que no existe una cultura de servicios extendida en el entorno de la distribución cerámica, ya que más del $40 \%$ de los encuestados cuenta con una gama de servicios complementarios compuesta por 2 o menos de estos. Esta conclusión fue corroborada durante la investigación cualitativa ya que los distribuidores nos manifestaron durante las entrevistas realizadas que no consideran al cliente final como un actor decisivo para la toma de decisiones de compra o incluso para propiciar adaptaciones al mercado.

No obstante, teniendo en cuenta los resultados esbozados no podemos rechazar la segunda hipótesis y encontramos una relación positiva entre el mayor nivel de relación entre fabricantes y distribuidores y la prestación de una gama de servicios complementarios más intensa a los clientes finales por parte de estos últimos.

Seguidamente, la tercera hipótesis propuesta relacionada con la aplicación de herramientas de medición de las percepciones del cliente fue igualmente sometida a tablas de contingencia para verificar si existe relación de causalidad alguna entre el nivel de valoración del intercambio entre fabricantes y distribuidores y la aplicación de las mismas.

En primer lugar, verificamos esta hipótesis teniendo en cuenta la aplicación de encuestas a los clientes, análisis que nos arroja en primer lugar la casi ausencia total de este tipo de herramientas para los encuestados. Sin embargo, los mejores resultados se producen nuevamente en el grupo que mejor valora a los proveedores, lo que nos permite basándonos en el resultado de la prueba chi-cuadrado $\mathrm{p}<0,05$, corroborar nuestra tercera hipótesis.

Para el caso de la realización de entrevistas con los clientes, los resultados son completamente opuestos, ya que aunque los porcentajes muestran que se realizan con mayor frecuencia que la aplicación de encuestas, los grupos de menor valoración de la relación son los que presentan mejores resultados. Estas diferencias son catalogadas como significativas por las pruebas de chi-cuadrado que muestran resultados al nivel $p<0,01$.

Sin embargo, si comparamos esta información con la obtenida en la investigación cualitativa que realizamos paralelamente a la aplicación de las encuestas, estos resultados no nos sorprenden ya que para los distribuidores la realización, de entrevistas obedece más a un intercambio de tipo informal llevado a cabo prácticamente con el día a día de las ventas que aun instrumento formalizado y sistematizado, lo qué bajo nuestra perspectiva limita sensiblemente los resultados obtenidos.

Ala luz de estos resultados, no es posible aceptar totalmente la tercera hipótesis propuesta y sería necesaria la obtención de más datos que nos permitan corroborar lo obtenido hasta el momento en este apartado que relaciona la aplicación de 
herramientas más formales de medición de las percepciones del cliente con los grupos que presentan una mejor relación con sus proveedores.

\section{CONCLUSIONES}

El presente trabajo aporta, por una parte un análisis del perfil de un sub sector, el de distribución de material cerámico, hasta ahora muy poco estudiado. Por otra, introduce una visión nueva que enlaza el distribuidor, tanto con fabricantes como con clientes finales, identificando los factores que inciden en el impacto que las relaciones mencionadas pueden tener en la creación de valor en la cadena.

Del análisis de los resultados del estudio estadístico se deduce que los tres factores mencionados: Actividades de orientación al mercado; intercambio de información estratégica con fabricantes; y servicios complementarios ofrecidos al cliente final conducen a la identificación y clasificación de las empresas analizadas en tres grupos con determinadas características de desempeño e impacto económico confirmando por lo tanto las hipótesis de partida. Así se concluye que el nivel de relación entre fabricantes y distribuidores tiene un efecto positivo sobre la realización de actividades orientadas al mercado/cliente por parte de los distribuidores así como sobre la intensidad de la gama de los servicios complementarios ofrecidos al cliente

Los datos analizados por los autores apuntan hacia la existencia de una dinámica de evolución en la distribución con una mayor aportación de valor por parte de la distribución. El análisis de las bases de datos económicas subraya que los distribuidores líderes tienen una mayor performance económica. Por su parte, los fabricantes líderes están desarrollando su propia red de distribución. En este sentido, cabe apuntar como ejemplo de la dinámica del sector distribuidor el mercado británico donde los distribuidores suponen la mayoría y constituyen un importante lobby. Aquí también se confirma la tendencia a proveer cada vez más servicios a los clientes finales.

\section{REFERENCIAS}

1. W.M. Lassar, J.L. Kerr, Strategy and Control in Supplier-Distributor Relationships: An Agency Perspective, Strategic Management Journal, 17, 8, 613-632 (1996).

2. R.J. Fontenot, R.J. Wilson, Relational exchange: A review of selected models for a prediction matrix of relationship activities, Journal of Business Research, 39, 1, 5-12 (1997).
3. C. Grönroos, Value-driven Relational Marketing: from Products to Resources and Competencies, Journal of Marketing Management, 13, 5, 407-419 (1997).

4. E. Gummesson, Implementation Requires a Relationship Marketing Paradigm, Journal of the Academy of Marketing Science, 26, 3, 242-249 (1998).

5. P.J. Kaufmann, R.P. Dant, The Dimensions of Commercial Exchange, Marketing Letters, 3, 2, 171-185, (1992).

6. J.L. Johnson, Strategic Integration in Industrial Distribution Channels: Managing the Interfirm Relationship as a Strategic Asset, Journal of the Academy of Marketing Science, 27, 1, 4-18 (1999).

7. S. González, Relationship Marketing and Vertical Constraints in the Distribution Channel, Non published Doctoral Thesis, Universidad de Oviedo (2001).

8. W.G. Rowe, J.G. Barnes, Relationship marketing and sustained competitive advantage". Journal of Marketing Focused Management, 281-97 (1998).

9. J. Anderson, Relationships in Business Markets: Exchange Episodes, Value Creation and Their Empirical Assessment, Journal of the Acad of Marketing Science, 23, 346-350 (1995).

10. A. Sharma, N. Tzokas, M. Saren, P. Kyziridis, Antecedents and Consequences of Relationship Marketing, Industrial Marketing Management, 28,3, 601-611 (1999).

11.0 B. Weitz, D. Sandy, Relationship Marketing and Distribution Channels, Journal of the Academy of Marketing Science. 23, 4, 305-320 (1995).

12. P. Simpson, J. Siguaw, T. Backer. A model of value creation. Suppliers behaviors and their impact on reseller-perceived value, Ind. Marketing Management ,30, 119-134 (2001).

13. S. F.; Slater, J. C. Narver, Market Orientation, Customer Value, and Superior Performance, Business Horizons, 37, 2, 22-28 (1994)

14. S. Deng, J. Dart, Measuring Market Orientation: A Multi-factor, Multi-item Approach, Journal of Marketing Management, 10, 8, 725-742 (1994).

15. R.F. Lusch, G.R. Laczniak, The evolving marketing concept, competitive intensity, and organizational performance. Journal of the Acad of Marketing Science, 15, 3, 1-11 (1987).

16. C. Grönroos, From Marketing Mix to Relationship Marketing: Towards a Paradigm Shift in Marketing", Asia-Australia Marketing Journal, 2, 1, 9-29 (1994).

17. C. Homburg, B. Garbe, Towards an Improved Understanding of Industrial Services:Quality Dimensions and Their Impact on Buyer-Seller Relationships, Journal of Business-to-Business Marketing, 6, 2, 39-71 (1999).

18. C. Narver, S.F. Slater, The Effect of a Market Orientation on Business Profitability, Journal of Marketing, 54, 4, 20-35 (1990).

19. J.A. Siguaw, P.M. Simpson, T.L. Baker, Effects of Supplier Market Orientation on Distributor Market Orientation and the Channel Relationship: The Distributor Perspective, Journal of Marketing, 62, 3, 99-111 (1998).

20. J. Albors, J.L. Hervás, La industria cerámica europea en el siglo XXI : Retos tecnológicos y desafíos de la próxima década, Bol. Soc. Esp. Cerám. V., 45, 4, 13-21 (2006).

21. J. Albors, P.B. Marquez, J.L. Hervás, Adopción contingente de tecnología de producción en el sector cerámico español. Un estudio empírico. Boletín Bol. Soc. Esp. Cerám. V., 45, 4, 24-36 (2006).

22. B. Segura, E. Vallada, C. Maroto, R. Ruiz, Análisis del sistema de operaciones en empresas del sector cerámico español, Bol. Soc. Esp. Cerám. V. 43, 6, 929-932 (2004).

23. E. Criado, M. Regueiro, E. Sánchez, La industria cerámica en España (19902000), Bol. Soc. Esp. Cerám. V., 40, 6, 413-428 (2001).

24. J. Franco, J., Souto, A., Rey, P. Guitián, F. Martínez Ínsua A., (2006), Contingent adoption of production technology in the Spanish tile ceramic cluster. An empirical study, Bol. Soc. Esp. Cerám. V., 40, 6, 338-347.

25. J.L. Hervás, Heterogeneidad Estratégica en un cluster. Evidencia empírica de la identificación de grupos estratégicos a través de la cadena de valor y su impacto en la performance en el sector industrial cerámico, Non published Doctoral Thesis, Universidad Politécnica de Valencia (2004). 